\title{
Tricetin, a dietary flavonoid, suppresses benzo(a)pyrene-induced human non-small cell lung cancer bone metastasis
}

\author{
JEN-YU HUNG ${ }^{1,2 *}$, WEI-AN CHANG ${ }^{2,3 *}$, YING-MING TSAI ${ }^{2,4}$, YA-LING HSU $^{4}$, \\ HUNG-HSING CHIANG ${ }^{3,5}$, SHAH-HWA CHOU ${ }^{5}$, MING-SHYAN HUANG ${ }^{1,2}$ and PO-LIN KUO ${ }^{3,6,7}$ \\ ${ }^{1}$ School of Medicine, College of Medicine, Kaohsiung Medical University, Kaohsiung 807; ${ }^{2}$ Division of Pulmonary and \\ Critical Care Medicine, Kaohsiung Medical University Hospital, Kaohsiung 807; ${ }^{3}$ Institute of Clinical Medicine and \\ ${ }^{4}$ Graduate Institute of Medicine, College of Medicine, Kaohsiung Medical University, Kaohsiung 807; ${ }^{5}$ Division of \\ Chest Surgery, Department of Surgery, Kaohsiung Medical University Hospital, Kaohsiung 807; ${ }^{6}$ Research \\ Center for Environmental Medicine, Kaohsiung Medical University, Kaohsiung 807; ${ }^{7}$ Institute of Medical \\ Science and Technology, National Sun Yat-Sen University, Kaohsiung 804, Taiwan, R.O.C.
}

Received December 23, 2014; Accepted February 6, 2015

DOI: 10.3892/ijo.2015.2915

\begin{abstract}
This is the first study to demonstrate that benzo(a)pyrene (BaP) was able to enhance the production of parathyroid hormone-related protein (PTHrP) by human non-small cell lung cancer H460 cells. Such effect would further contribute to bone metastasis of lung cancer by increasing osteoclastogenesis. This study is also the first to reveal that tricetin (TCN), a flavonoid derivative found in Myrtaceae pollen and Eucalyptus honey, was able to reverse BaP-mediated bone resorption activity of lung cancer cells. Human non-small cell lung cancer H460 cells were treated with $\mathrm{BaP}$ to generate conditioned medium. When osteoblasts were cultured with BaP-H460-CM, their expression of osteoclastogenesis activator macrophage colony-stimulating factor (M-CSF) and receptor activator of nuclear factor $\kappa \mathrm{B}$ ligand (RANKL) was increased. BaP-H460-CM reduced the production of osteoprotegerin (OPG), an osteoclastogenesis inhibitor, in osteoblasts. Osteoclastogenesis and bone resorption activity of $\mathrm{H} 460$ cells were increased by BaP-H460-CM. With BaP-mediated PTHrP upregulation, IL-8 secretion in H460 cells was increased contributing to human non-small cell lung cancer-mediated osteoclast differentiation and
\end{abstract}

Correspondence to: Professor Ming-Shyan Huang, School of Medicine, College of Medicine, Kaohsiung Medical University, No. 100 Shih-Chuan 1st Road, Kaohsiung 807, Taiwan, R.O.C.

E-mail: shyan310@gmail.com

Professor Po-Lin Kuo, Institute of Clinical Medicine, College of Medicine, Kaohsiung Medical University, No. 100 Shih-Chuan 1st Road, Kaohsiung 807, Taiwan, R.O.C.

E-mail: kuopolin@seed.net.tw

*Contributed equally

Key words: tricetin, benzo(a)pyrene, PTHrP, osteoclastogenesis bone resorption. Moreover, TCN suppressed BaP-mediated bone resorption. Therefore, TCN may be a novel agent for treatment of non-small cell lung cancer patients with bone metastasis.

\section{Introduction}

Lung cancer is one of the common cancers in the world, and also one of the leading causes of cancer-related deaths worldwide (1-3). Lung cancer metastasizes to the skeletal system frequently. About 30-40\% of lung cancer patients will develop bone metastasis during the progression of their disease, which results significant impact on the patients' quality of life, morbidity and survival (3-5). Bone metastasis from lung cancer usually causes osteolytic lesions characterized by increased osteoclast activity $(3,4)$ and decreased osteoblast capacity (6-8). Parathyroid hormone-related protein (PTHrP), produced by lung cancer cells, will stimulate osteoblasts to express elevated levels of receptor activator of nuclear factor $\kappa \mathrm{B}$ ligand (RANKL) and will stimulate osteoclastogenesis by binding to the receptor RANK and activating its downstream signaling pathways in hematopoietic osteoclast precursors $(3,9)$. Thus, therapy targeting osteoclast/osteoblast interactions during lung cancer progression isimportant.

Polycyclic aromatic hydrocarbons (PAHs), are formed by the incomplete combustion of organic matter. Benzo(a) pyrene $(\mathrm{BaP})$ is the most commonly measured and studied PAH. They usually present in the environment at detectable levels in many types of uncooked food, and cooking process could generate PAHs in food. Several studies have been conducted to determine the levels of exposure to PAHs from representative human diet and the proportion of the overall burden of environmental exposure to PAHs that is attributable to diet (10-13). Previous studies demonstrated the association between PAHs and an increased risk of respiratory tract cancer (14-16). Exposure to BaP enhances the invasion and metastasis of lung cancer cells and BALB/c $3 \mathrm{~T} 3$ cells in vivo and in vitro (17-19). Moreover, BaP can enhance the expression 
level of epithelial-mesenchymal transition-related genes (20) and can promote migration and invasion of lung cancer cells through upregulating Twist (21).

Tricetin (TCN) (5,7,3',4',5'-pentahydroxyflavone), a flavonoid derivative found in Myrtaceae pollen and Eucalyptus honey (22-24), possesses potent anti-inflammatory and anti-cancer activities (25-27). This study evaluated the effects of $\mathrm{BaP}$ in human non-small cell lung cancer bone metastasis and investigated the potential role of TCN against the effects from $\mathrm{BaP}$ on human non-small cell lung cancer.

\section{Materials and methods}

Chemicals. TCN was obtained from Extrasynthese (Genay, France), dissolved in dimethyl sulfoxide (DMSO) (SigmaAldrich, St. Louis, MO, USA), and stored at $-20^{\circ} \mathrm{C}$. Control cultures received the carrier solvent (0.1\% DMSO). All chemicals used were in their purest form available commercially.

Cell culture and conditioned medium. Human non-small cell lung cancer $\mathrm{H} 460$ cells were obtained from the American Type Culture Collection (HTB-177) (Manassas, VA, USA) and cultured in RPMI-1640 medium containing 10\% fetal bovine serum (FBS) (both from Gibco-BRL, Gaithersburg, MD, USA). Human primary osteoblasts were obtained from Lonza (Walkersville, MD, USA) and cultured in osteoblast medium (OBM) (Lonza).

To obtain the various conditioned media (CM), H460 cells ( $2 \times 10^{6} / 100 \mathrm{~mm}$ dish) were treated with various concentrations of $\mathrm{BaP}$ (Sigma-Aldrich) for $6 \mathrm{~h}$. After treatment, the medium was replaced and the supernatant harvested and filtered $(0.22 \mathrm{~mm})$ after $24 \mathrm{~h}$ of incubation.

Measurement of secreted factors. Supernatants from osteoblasts and $\mathrm{H} 460$ cells were collected. Levels of osteoprotegerin (OPG), macrophage colony-stimulating factor (M-CSF), RANKL and IL-8 were assessed and quantified using the DuoSet enzyme-linked immunosorbent assay (ELISA) (R\&D Systems, Minneapolis, MN, USA). PTHrP levels were determined by an ELISA kit (Abnova Corp., Taipei, Taiwan).

Isolation of $\mathrm{CD}^{+} 4^{+}$monocytes and osteoclast differentiation. Monocytes were purified from peripheral blood mononuclear cells (PBMCs) obtained from healthy donors. Mononuclear cells were isolated from blood by Ficoll-Hypaque gradient (GE Healthcare UK, Ltd., Buckinghamshire, UK). CD14+ monocytes were purified using $\mathrm{CD} 14^{+}$monoclonal antibody-conjugated magnetic beads (MACS MicroBeads; Miltenyi Biotec $\mathrm{GmbH}$, Bergisch Gladbach, Germany), according to the manufacturer's instructions. Osteoclasts were generated by culturing $\mathrm{CD}_{1} 4^{+}$monocytes in medium containing $20 \%$ vehicle control-CM-cultured osteoblasts or BaP-H460-CM-cultured osteoblasts presented in $100 \mathrm{ng} / \mathrm{ml}$ M-CSF and $50 \mathrm{ng} / \mathrm{ml}$ RANKL (R\&D Systems) for 14-21 days. The medium was replaced with fresh medium containing M-CSF and RANKL every 5 days.

Osteoclast formation was measured by quantifying cells positively stained by TRAP (Sigma-Aldrich). Osteoclasts were deemed TRAP-positive by light microscopy that revealed staining of multinuclear ( $>3$ nuclei) cells. The TRAP-positive cells and the number of nuclei per TRAP-positive cells in each well were counted. The bone resorption activity of osteoclasts was assessed by a 48 -well plate bone resorption assay (Cosmo Bio Co., Ltd., Tokyo, Japan), under the same culture conditions as described above.

The Institutional Review Board (IRB) of Kaohsiung Medical University Chung-Ho Memorial Hospital (Kaohsiung, Taiwan) approved the study protocol and all of the participants provided written informed consent in accordance with the Declaration of Helsinki (IRB nos.: KMUH-IRB-990345, KMUH-IRB-20110377 and KMUH-IRB-20130054).

Real-time polymerase chain reaction $(R T-q P C R)$. The TRIzol reagent (Invitrogen Life Technologies, Carlsbad, CA, USA) was used for RNA isolation while cDNA was prepared using an oligo(dT) primer and reverse transcriptase (Takara Bio, Inc., Shiga, Japan) following standard protocols. The RT-qPCR was performed using SYBR-Green on the ABI 7500 Real-Time PCR System (Applied Biosystems, Foster City, CA, USA). Each PCR reaction mixture contained $200 \mathrm{nM}$ of each primer, $10 \mu 12 \mathrm{X}$ SYBR-Green PCR Master Mix (Applied Biosystems), and $5 \mu \mathrm{l}$ cDNA and RNase-free water for a total volume of $20 \mu \mathrm{l}$. The RT-qPCR was conducted with a denaturation step at $95^{\circ} \mathrm{C}$ for $10 \mathrm{~min}$, then for 40 cycles at $95^{\circ} \mathrm{C}$ for $15 \mathrm{sec}$, and $60^{\circ} \mathrm{C}$ for $1 \mathrm{~min}$. All PCRs were performed in triplicate and normalized to internal control glyceraldehyde-3-phosphate dehydrogenase (GAPDH) mRNA. The relative expression level was determined using the $2^{-\Delta \Delta \mathrm{CT}}$ method.

PTHrP knockdown. H460 cells were transfected with $20 \mathrm{nM}$ non-target or PTHrP siRNA pool (Dharmacon, Inc., Lafayette, CO, USA) by DharmaFECT 4 Transfection Reagents, according to the manufacturer's instructions. After $24 \mathrm{~h}$ transfection, the medium was changed to whole medium and the cells were treated with $\mathrm{BaP}$. The PTHrP changes were measured by RT-qPCR.

Statistical analysis. Data are expressed as means \pm standard errors. Statistical comparisons of the results were made using analysis of variance (ANOVA). Significant differences $(p<0.05)$ between the means of the test groups were analyzed by Student's t-test.

\section{Results}

BaP induces PTHrP secretion in human non-small cell lung cancer. To investigate the effects of $\mathrm{BaP}$ on PTHrP secretion by human non-small cell lung cancer cells, BaP was added to the culture medium of $\mathrm{H} 460$ cells to the concentration of $10 \mu \mathrm{M}$ for $6 \mathrm{~h}$. After washing, cells were cultured with new medium for another $24 \mathrm{~h}$, the conditioned medium of BaP-treated $\mathrm{H} 460$ (BaP-H460-CM) was harvested and PTHrP levels of these $\mathrm{CM}$ were assessed. After exposure to $\mathrm{BaP}$, the production of PTHrP in human non-small cell lung cancer H460 cells was increased (Fig. 1A). H460 cells were also treated with different concentrations of $\mathrm{BaP}$, the result revealed that $\mathrm{BaP}$ increased the production of PTHrP in $\mathrm{H} 460$ cells in a dose-dependent manner (Fig. 1B). 

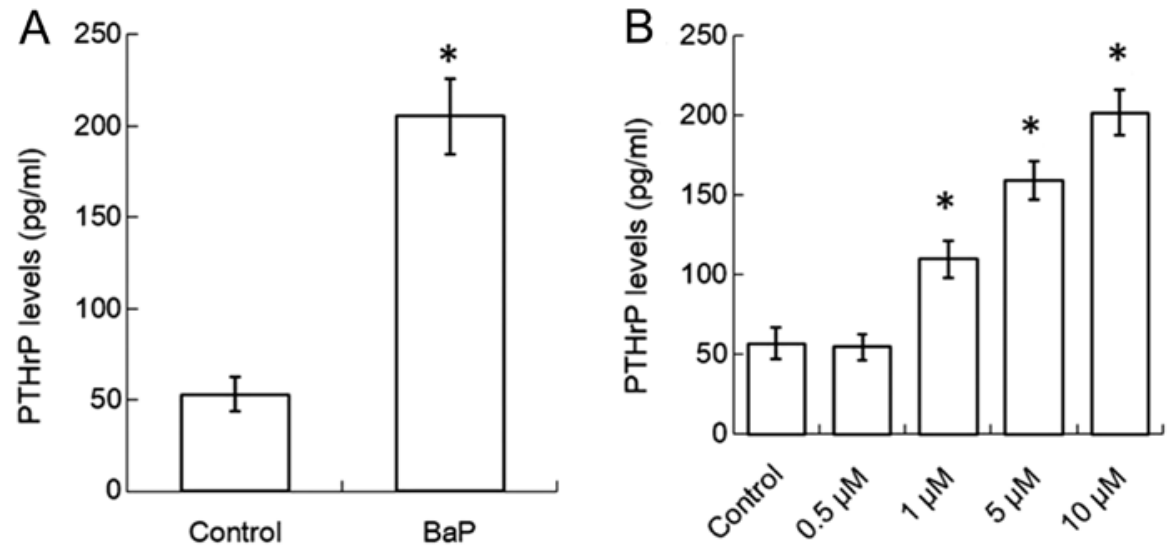

Figure 1. Benzo(a)pyrene (BaP) increased parathyroid hormone-related protein (PTHrP) expression in human non-small cell lung cancer. (A) The effects of $10 \mu \mathrm{M} \mathrm{BaP}$ on PTHrP levels in $\mathrm{H} 460$ cells. (B) BaP increased PTHrP expression in a dose-dependent manner. $\mathrm{H} 460$ cells treated with $10 \mu \mathrm{M}$ BaP or various concentrations of $\mathrm{BaP}$ for $6 \mathrm{~h}$. After washing and a 24-h culture, the conditioned media (CM) of BaP-treated H460 cells (BaP-H460-CM) were harvested, and PTHrP levels in these CM were then assessed by enzyme-linked immunosorbent assay (ELISA). Each value was the mean \pm SD of three independent experiments. ${ }^{*} \mathrm{P}<0.05$, significant difference between the control and test groups.

A

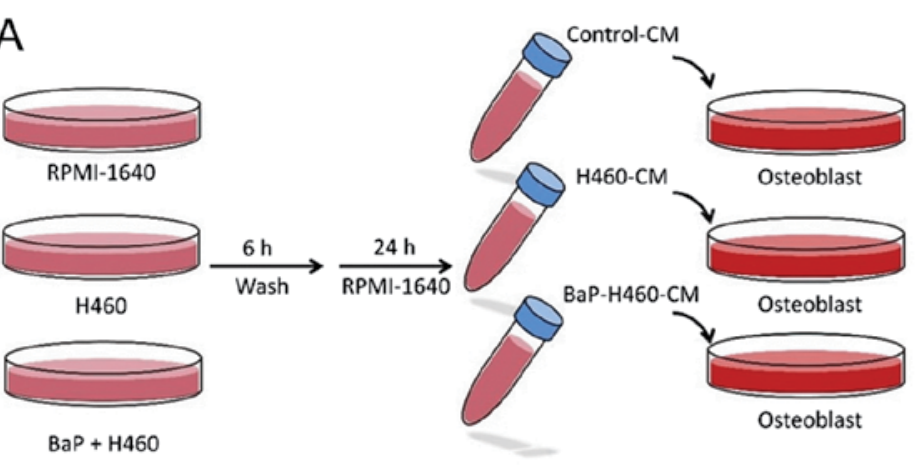

$\mathrm{BaP}+\mathrm{H} 460$

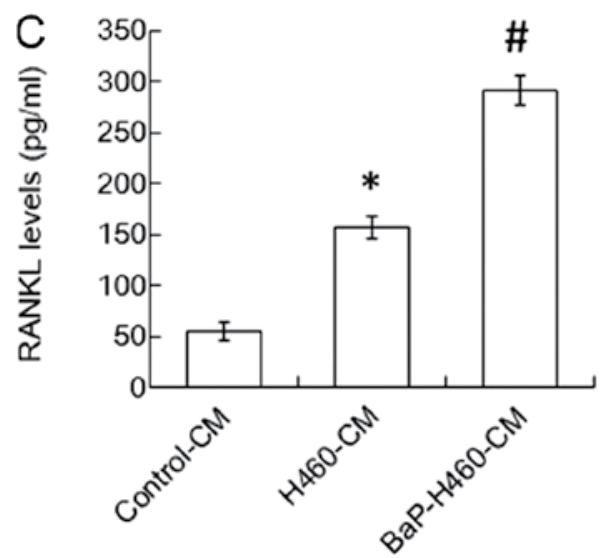

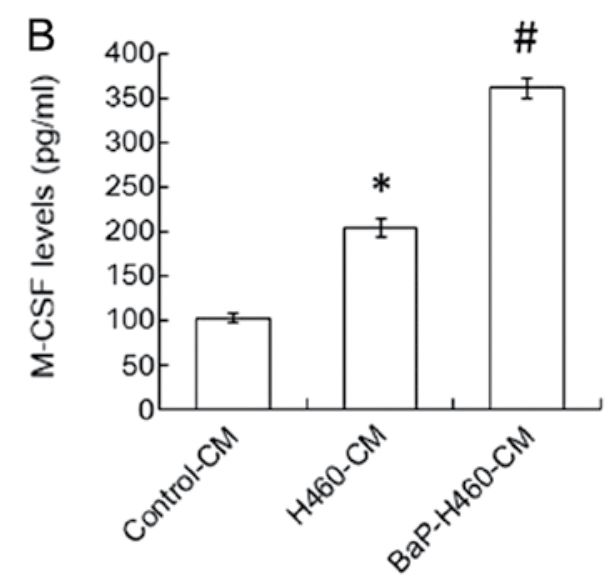

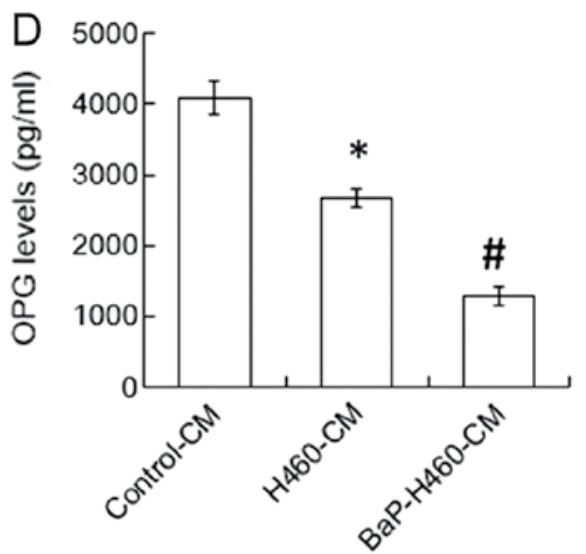

Figure 2. Benzo(a)pyrene (BaP) reinforces the interference of human non-small cell lung cancer in osteoblasts. (A) Flow chart of the production of control-CM, $\mathrm{H} 460-\mathrm{CM}$, and BaP-H460-CM. BaP $(10 \mu \mathrm{M})$ enhanced the stimulatory effect of $\mathrm{H} 460$ cells on the expression of (B) macrophage colony-stimulating factor (M-CSF) and (C) receptor activator of nuclear factor $\kappa \mathrm{B}$ ligand (RANKL) in osteoblast. (D) BaP (10 $\mu \mathrm{M}$ ) potentiated the inhibitory effect of human non-small cell lung cancer on osteoprotegerin (OPG) expression in osteoblasts. $\mathrm{H} 460$ cells treated with or without $10 \mathrm{mM} \mathrm{BaP}$ for $6 \mathrm{~h}$. After washing and a 24-h culture, the conditioned media (CM) of non-treated or BaP-treated $\mathrm{H} 460$ cells ( $\mathrm{H} 460-\mathrm{CM}$ and BaP-H460-CM) were harvested and stored at $-80^{\circ} \mathrm{C}$. Osteoblasts were treated with various CM for $24 \mathrm{~h}$. The levels of M-CSF, RANKL or OPG in the supernatants of osteoblasts were assessed by enzyme-linked immunosorbent assay (ELISA). Each value was the mean \pm SD of three independent experiments. "Significant difference with control-CM treatment, "significant difference with H460-CM treatment, $\mathrm{p}<0.05$. 

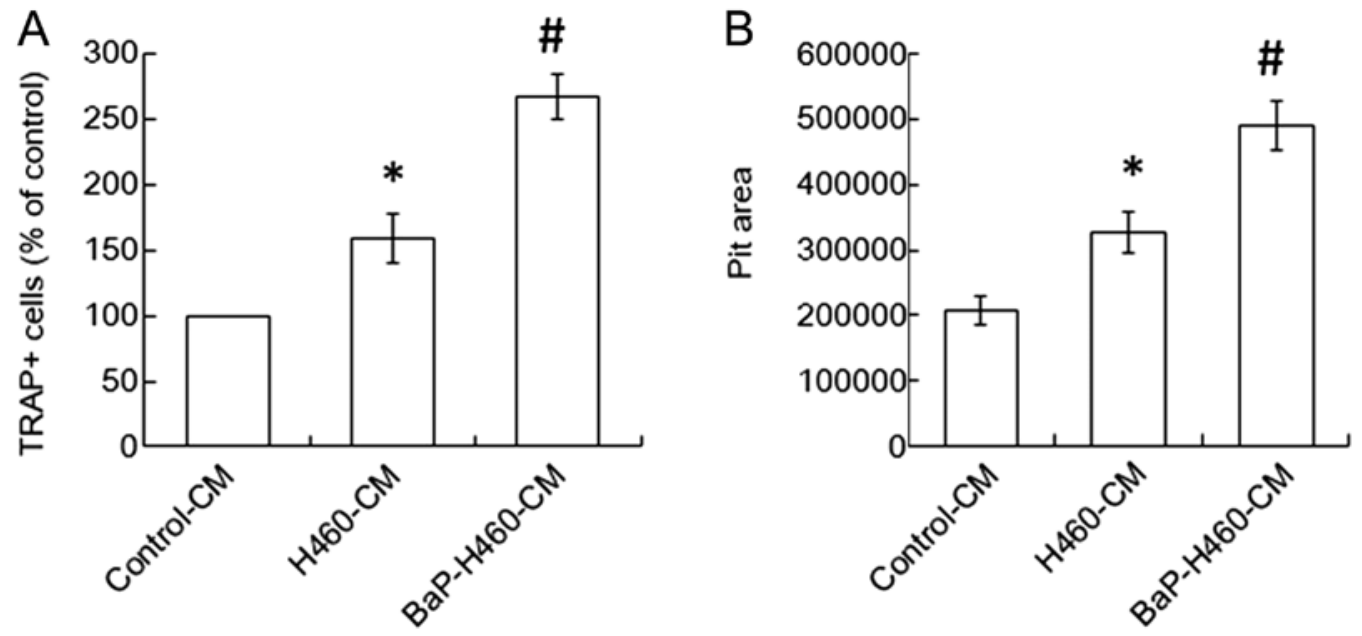

Figure 3. Benzo(a)pyrene (BaP) strengthens the stimulatory effect of human non-small cell lung cancer on osteoclastogenesis and bone resorption. (A) BaP $(10 \mu \mathrm{M})$ increased the effect of $\mathrm{H} 460$ cells on osteoclastogenesis and (B) their bone resorption activity. Osteoclastogenesis was determined by TRAP staining (osteoclast: $>3$ nuclei, TRAP-positive). CD14 ${ }^{+}$monocytes were treated with H460-CM and BaP-H460-CM (20\%) cultured with macrophage colony-stimulating factor (M-CSF) and receptor activator of nuclear factor $\kappa B$ ligand (RANKL) for 14-21 days. Media were replaced every 5 days. Osteoclasts were then stained by TRAP. Multinucleated ( $>3$ nuclei) TRAP-positive cells are defined as osteoclasts. The pit area was determined by AlphaEaseFC software. Each value was the mean \pm SD of three independent experiments. "Significant difference with control-CM treatment, "significant difference with H460-CM treatment, $\mathrm{p}<0.05$.
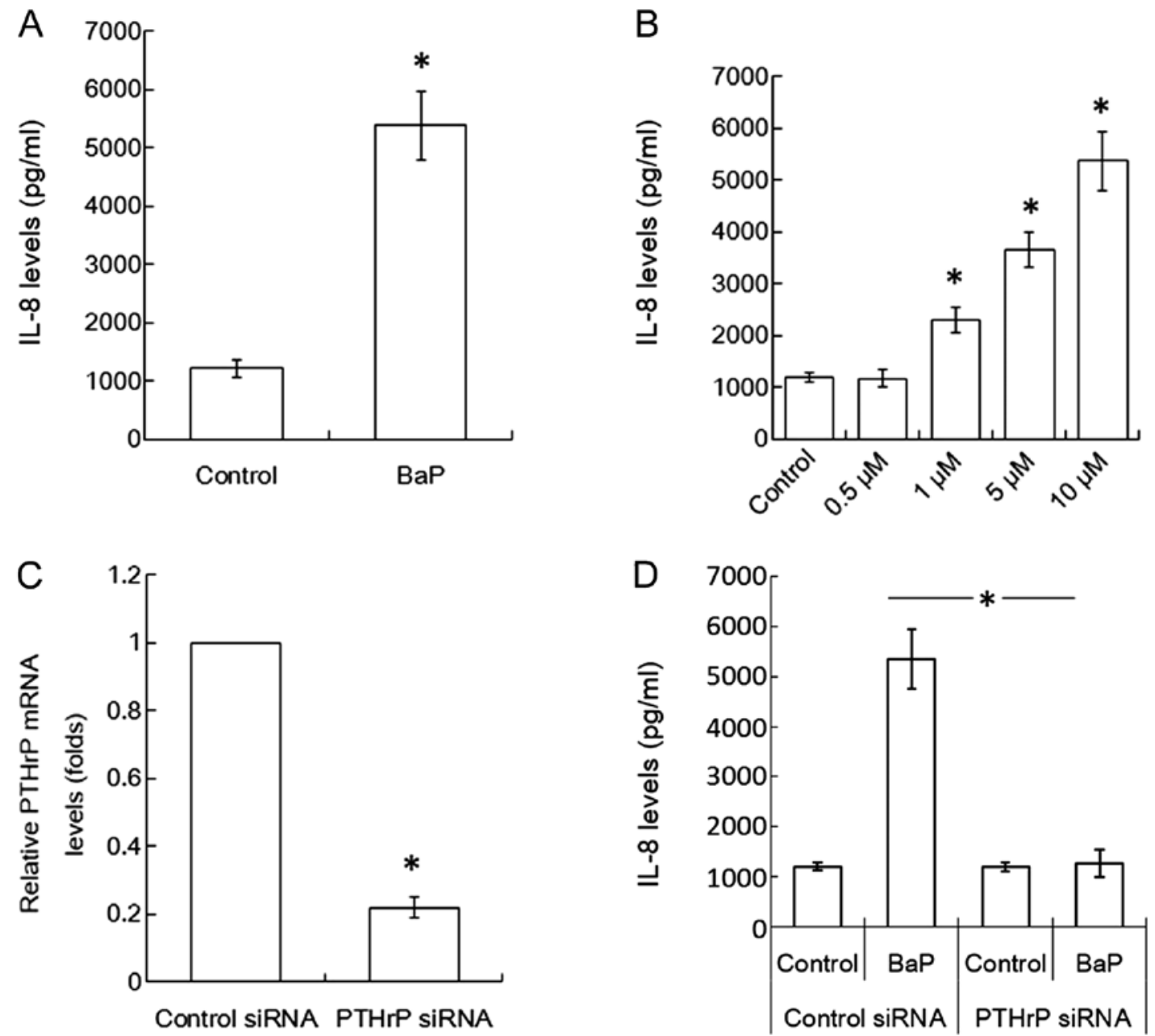

Figure 4. Benzo(a)pyrene (BaP) upregulates the expression of IL-8 by increasing parathyroid hormone-related protein (PTHrP) in human non-small cell lung cancer. (A) The effect of $10 \mu \mathrm{M}$ BaP on IL-8 levels in H460 cells. (B) BaP upregulated IL-8 expression in a dose-dependent manner. (C) The efficiency of PTHrP siRNA transfection. (D) Inhibition of PTHrP decreased the upregulation of IL-8 by BaP in H460 cells. The IL-8 levels were assessed by enzyme-linked immunosorbent assay (ELISA), and the efficacy of siRNA was determined by real-time polymerase chain reaction (RT-qPCR). H460- or siRNA-transfected $\mathrm{H} 460$ cells treated with $\mathrm{BaP}(10 \mu \mathrm{M}$ or various concentrations) for $24 \mathrm{~h}$. PTHrP levels were determined by ELISA. Each value is the mean \pm SD of three independent experiments. ${ }^{*} \mathrm{P}<0.05$, significant difference between the control and test groups. 
and RANKL)/inhibitor (OPG) produced by osteoblasts $(28,29)$. To determine if $\mathrm{BaP}$ influences the interaction between non-small cell lung cancer cells and the secretion of M-CSF, RANKL, and OPG by osteoblasts, H460 cells were treated with $0.1 \%$ DMSO or $10 \mu \mathrm{M}$ BaP for $6 \mathrm{~h}$. Then $\mathrm{BaP}$ was removed by washing. H460 cells were cultured with fresh medium and the culture medium was collected thereafter (defined as control-CM, H460-CM, and BaP-H460-CM).

Human osteoblasts were cultured with the $\mathrm{CM}$ prepared to assess the effects of $\mathrm{BaP}$ on the interaction between non-small cell lung cancer and osteoblasts (Fig. 2A). H460-CM was found to be able to increase the M-CSF and RANKL expressions in osteoblasts and such stimulatory effect was further enhanced when $\mathrm{H} 460$ cells were pre-treated with $\mathrm{BaP}$ (Fig. 2B and C). In contrast, H460-CM decreased the OPG expression in osteoblasts and this inhibitory effect was strengthened when non-small cell lung cancer cells were exposed to BaP (Fig. 2D).

BaP increased human non-small cell lung cancer $H 460$ cell-mediated osteoclastogenesis and bone resorption. To assess the effect of $\mathrm{BaP}$ on non-small cell lung cancer-mediated osteoclastogenesis, TRAP and bone resorption assays were tested. H460-CM increased osteoclastogenesis induced by H460 cells, and such effect was strengthened when H460 cells were pre-treated with BaP (Fig. 3A). Similarly, H460-CMs enhanced bone resorption activity and such enhancement further intensified once H460 cells were pre-treated with BaP (Fig. 3B).

PTHrP/IL-8 autocrine loop is involved in the stimulation of BaP on non-small cell lung cancer-mediated osteoclastogenesis. Since PTHrP was reported to be able to increase IL-8 expression of cancer cells (30), this study assessed whether BaP increased the effect of $\mathrm{H} 460$ cells on osteoclastogenesis is through the PTHrP/IL-8 loop or not. The level of IL-8 was higher in BaP-treated $\mathrm{H} 460$ cells than in the control group (Fig. 4A). Also, the effect of BaP to increase IL-8 expression from H460 cells was demonstrated to be in a dose-dependent (Fig. 4B).

To confirm the role of PTHrP in the upregulation of IL-8 induced by BaP, H460 cells were transfected with PTHrP siRNA. The expression of PTHrP mRNA in H460 cells was decreased $\sim 78 \%$ when the cells were transfected with PTHrP siRNA (Fig. 4C). PTHrP silencing prevented the upregulation effect from BaP on the expression of IL-8 in H460 cells (Fig. 4D).

TCN suppresses BaP-mediated bone resorption. To access the effects of TCN on BaP-mediated non-small cell lung cancer bone metastasis, BaP-induced PTHrP and IL- 8 secretion by non-small cell lung cancer $\mathrm{H} 460$ cells were tested again, both PTHrP and IL- 8 secretion were decreased by $1 \mu \mathrm{M}$ TCN treatment (Fig. 5A and B). Similarly, the CM of BaP-treated H460 cells enhanced RANKL upregulation in osteoblasts, and $1 \mu \mathrm{M}$ TCN treatment blocked such upregulation (Fig. 5C). The activity of TCN on human non-small cell lung cancer-mediated interaction of osteoblasts and osteoclasts was further investigated (Fig. 6A). Osteoclastogenesis and bone resorption were both significantly decreased by TCN treatment (Fig. 6B and C).

\section{Discussion}

Bone metastasis is a devastating event for lung cancer patients because once it occurs, the morbidity and mortality will increase $(31,32)$. Sone and Yano have demonstrated that several compounds, including bisphosphonates and reveromycin A, able to suppress the activity of osteoclast, are beneficial for the treatments of lung cancer patients with bone metastasis (33). However, 30-50\% of lung cancer patients still developed new bone metastasis or skeletal complications while they are receiving such therapy, which emphasizes the necessity for new therapies $(34,35)$. Evaluation of the differentiation of osteoclast is important in bone metastasis of lung cancer. Multimodality therapy is necessary to improve the efficacy of therapy against lung cancer bone metastasis (31-33). This is the first study to demonstrate that $\mathrm{BaP}$ increases the stimulatory effect of non-small cell lung cancer on osteoclastogenesis and osteoclastic bone resorption activity directly via PTHrP/IL- 8 and by interfering in the osteoblast-osteoclast interaction.

PTHrP, a potent activator of osteoclastic bone resorption, is an important pathologic factor for hypercalcemia among cancer patients (36-38). PTHrP stimulates osteoclastogenesis by increasing RANKL expression and by reducing OPG expression in osteoblasts. However, it does not act directly on the precursors of the osteoclasts $(39,40)$. RANKL binds with the RANK receptor of the precursors of osteoclasts and induces the formation of mature osteoclasts in the presence of M-CSF $(41,42)$.

OPG is a soluble decoy receptor for RANKL, with the ability to decrease osteoclastogenesis (43). Increase of RANKL/OPG ratio by cancer-derived PTHrP results in osteoclastic bone resorption $(3,44)$. This study shows that $\mathrm{BaP}$ upregulates the secretion of PTHrP in non-small cell lung cancer cells. Besides upregulation of PTHrP, BaP can reinforce the effects of non-small cell lung cancer on osteoblasts, including increased M-CSF and RANKL, and decreased OPG. These results suggest that $\mathrm{BaP}$ may worsen bone metastasis in non-small cell lung cancer.

Many studies have reported that non-small cell lung cancer expresses high levels of IL-8, which enhances both osteoclastogenesis and bone resorption $(45,46)$. PTHrP has been reported to enhance osteoclastogenesis by increasing the expression of osteoclast stimulatory factors such as IL-8 $(3,44)$. The present study demonstrates that BaP increases IL-8 expression in non-small cell lung cancer cells. Inhibition of PTHrP by siRNA transfection prevents the upregulation effects from BaP on IL-8 secretion. These results suggest that PTHrP is a major mediator involved in the stimulatory effect of BaP on IL- 8 production. Furthermore, BaP enhances the stimulatory effect of non-small cell lung cancer on osteoclastogenesis and their bone resorption activity. Thus, the regulation of IL- 8 expression by $\mathrm{BaP}$ through $\mathrm{PTHrP}$ is a key point in $\mathrm{BaP}$-induced osteoclastogenesis and bone resorption.

Current therapy for bone metastases have limited efficacy and are only palliative. The side-effects of these treatments, renal toxicity and osteonecrosis of the jaw potentially will decrease the quality of life of these lung cancer patients $(47,48)$. In addition, eliminating all $\mathrm{BaP}$ exposure may be impossible 

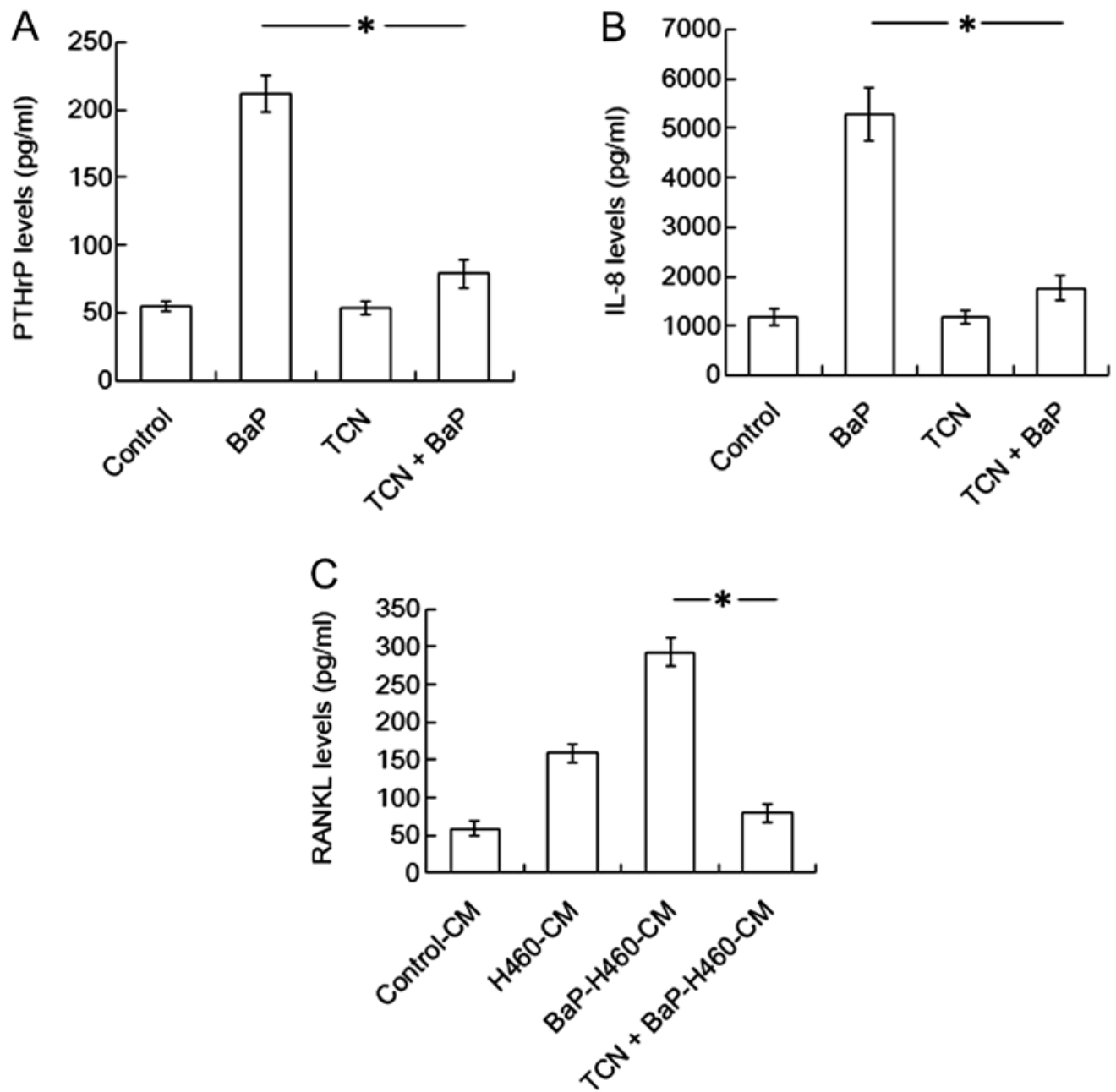

Figure 5. Tricetin (TCN) decreases the effects of benzo(a)pyrene (BaP) on parathyroid hormone-related protein (PTHrP), IL-8, and receptor activator of nuclear factor $\mathrm{\kappa B}$ ligand (RANKL) expression. TCN $(1 \mu \mathrm{M})$ decreased the upregulation of BaP on (A) PTHrP and (B) IL-8 in H460 cells. (C) TCN decreased the effects of $\mathrm{BaP}$ on RANKL expression of osteoblasts. For (A) and (B), $\mathrm{H} 460$ cells were pre-treated with TCN for $1 \mathrm{~h}$, then incubated with $\mathrm{BaP}(10 \mu \mathrm{M})$ for another $6 \mathrm{~h}$ for PTHrP, or $24 \mathrm{~h}$ for IL-8 analysis. After washing and a 24-h culture, the culture media were collected and PTHrP levels were assessed by enzyme-linked immunosorbent assay (ELISA). (C), H460 cells were pre-treated with TCN for $1 \mathrm{~h}$, then incubated with BaP (10 $\mu \mathrm{M})$ for another $6 \mathrm{~h}$. After washing and a 24-h culture, the culture media were collected, then added to osteoblasts for another $24 \mathrm{~h}$. The levels of RANKL in the supernatants of osteoblasts were assessed by ELISA. Each value is the mean $\pm \mathrm{SD}$ of three independent experiments. " $\mathrm{P}<0.05$, or significant difference between the control and test groups.

because BaP is widely present in modern life. It is therefore important that strategies be developed for preventing bone metastasis in non-small cell lung cancer.

Our data show that TCN, a flavonoid derivative found in Myrtaceae pollen and Eucalyptus honey, exhibits effects to decrease PTHrP expression in non-small cell lung cancer $\mathrm{H} 460$ cells. Simultaneously, TCN also decreases IL-8 expression, resulting in the inhibition of $\mathrm{H} 460$-mediated osteoclastogenesis and bone resorption. Moreover, TCN also decreases the stimulatory effect from non-small cell lung cancer on RANKL espression of osteoblasts, suggesting that TCN may be a potential agent to prevent the aggravating effect from $\mathrm{BaP}$ on non-small cell lung cancer bone metastasis.

In conclusion, there are two novel findings in this study: it is the first study to demonstrate that BaP increases the stimulatory effect of human non-small cell lung cancer on osteoclastogenesis and their activity of osteoclast bone resorption directly by PTHrP/IL- 8 and by interfering in the osteoblast-osteoclast interaction, and it is also the first to reveal that TCN, a flavonoid derivative found in Myrtaceae pollen and Eucalyptus honey, reverses BaP-mediated bone resorption (Fig. 7).

\section{Acknowledgements}

This study was supported by grants from the National Science Council (NSC 101-2628-B-037-001-MY3; NSC 101-2320-B-037-043-MY3), the Ministry of Science and Technology (MOST 103-2320-B-037-006-MY3; MOST 1032314-B-037-052; MOST 103-2320-B-037-032), the Kaohsiung Medical University 'Aim for the Top 500 Universities Grant, Grant no. KMU-DT103008', the Kaohsiung Medical University 'Aim for the Top Universities Grant, Grant nos. KMU-TP103A19 and KMU-TP103A20', and the Kaohsiung Medical University Hospital Research Foundation (KMUH101-1M08). The authors thank the Center for Research Resources and Development of Kaohsiung Medical University for its support with the instrumentation. 
A
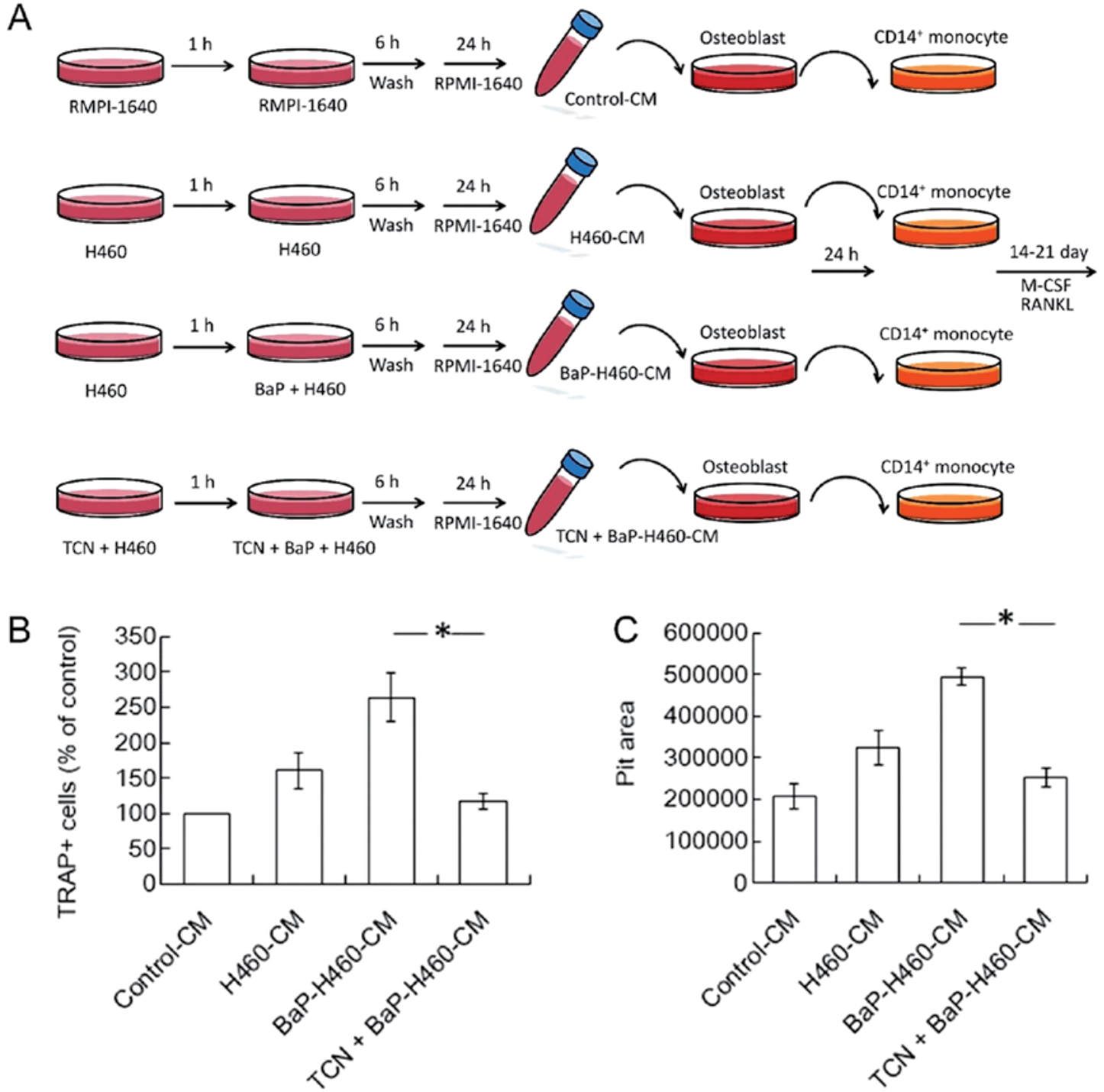

Figure 6. Tricetin (TCN) decreased the effects of benzo(a)pyrene (BaP) on human non-small cell lung cancer bone metastasis events. (A) Flow chart of production of control-CM, H460-CM, BaP-H460-CM, TCN + BaP-H460-CM or various osteoblast conditioned media (CM). TCN decreased the effects of BaP on (B) osteoclastogenesis and (C) bone resorption. For (B) and (C), $\mathrm{H} 460$ cells were pre-treated with TCN for $1 \mathrm{~h}$, then incubated with BaP (10 $\mu \mathrm{M})$ for another $6 \mathrm{~h}$. After washing and a $24-\mathrm{h}$ culture, the culture medium (20\%) was collected, then added to CD14 ${ }^{+}$monocytes presenting in macrophage colony-stimulating factor (M-CSF) and receptor activator of nuclear factor $\kappa B$ ligand (RANKL) for a 14-21 days. The osteoclastogenesis and bone resorption was conducted as described above. Each value is the mean $\pm \mathrm{SD}$ of three independent experiments. ${ }^{*} \mathrm{P}<0.05$, or significant difference between the control and test groups.
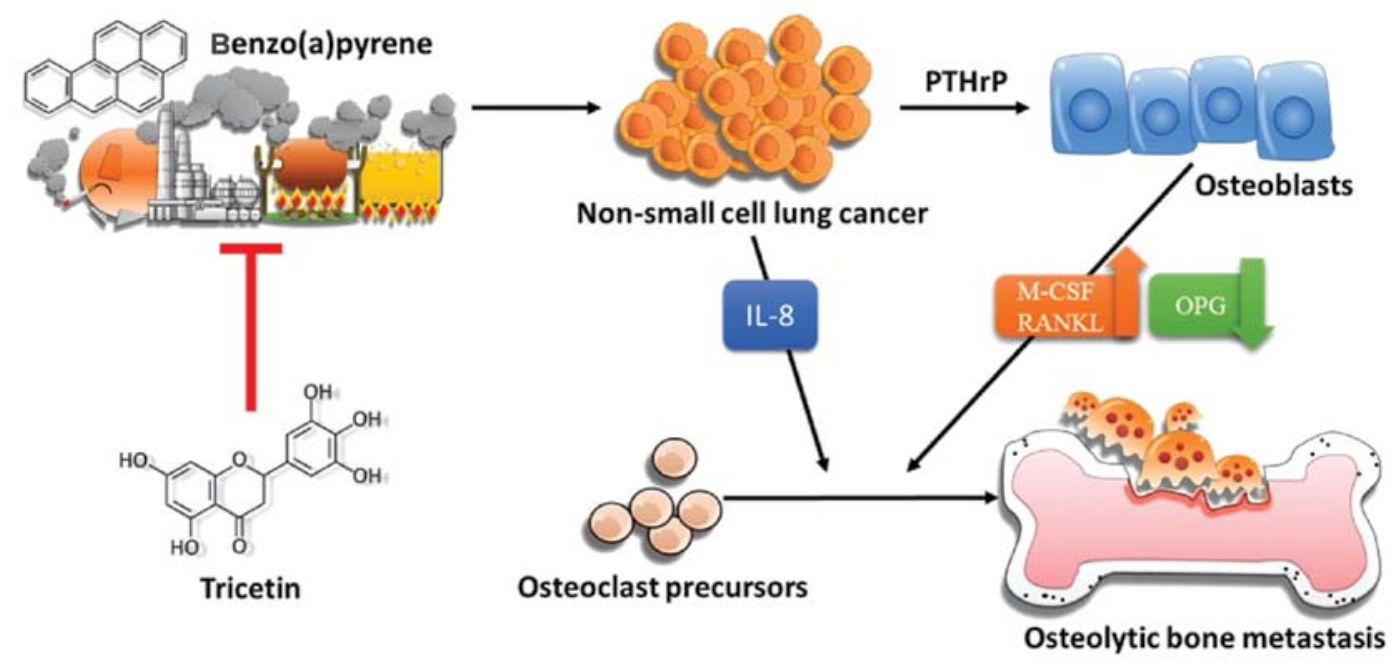

Figure 7. Scheme of proposed tricetin (TCN)-inhibited benzo(a)pyrene (BaP)-induced human non-small cell lung cancer bone metastasis. 


\section{References}

1. Siegel R, Ward E, Brawley O and Jemal A: Cancer statistics, 2011: The impact of eliminating socioeconomic and racial disparities on premature cancer deaths. CA Cancer J Clin 61: 212-236, 2011.

2. Zükin M: Epidermal growth factor receptor inhibitors in non-small cell lung cancer: Current status and future perspectives. Rev Assoc Med Bras 58: 263-268, 2012.

3. Kuo PL, Liao SH, Hung JY, Huang MS and Hsu YL: MicroRNA-33a functions as a bone metastasis suppressor in lung cancer by targeting parathyroid hormone related protein. Biochim Biophys Acta 1830: 3756-3766, 2013.

4. Al Husaini H, Wheatley-Price P, Clemons M and Shepherd FA: Prevention and management of bone metastases in lung cancer: A review. J Thorac Oncol 4: 251-259, 2009.

5. Hirsh V, Major PP, Lipton A, Cook RJ, Langer CJ, Smith MR, Brown JE and Coleman RE: Zoledronic acid and survival in patients with metastatic bone disease from lung cancer and elevated markers of osteoclast activity. J Thorac Oncol 3: 228-236, 2008.

6. Sterling JA, Edwards JR, Martin TJ and Mundy GR: Advances in the biology of bone metastasis: How the skeleton affects tumor behavior. Bone 48: 6-15, 2011.

7. Sims NA and Gooi JH: Bone remodeling: Multiple cellular interactions required for coupling of bone formation and resorption. Semin Cell Dev Biol 19: 444-451, 2008.

8. Miller RE, Jones JC, Tometsko M, Blake ML and Dougall WC RANKL inhibition blocks osteolytic lesions and reduces skeletal tumor burden in models of non-small-cell lung cancer bone metastases. J Thorac Oncol 9: 345-354, 2014.

9. Esposito $\mathrm{M}$ and Kang Y: Targeting tumor-stromal interactions in bone metastasis. Pharmacol Ther 141: 222-233, 2014.

10. Phillips DH: Polycyclic aromatic hydrocarbons in the diet. Mutat Res 443: 139-147, 1999.

11. Anastasio A, Mercogliano R, Vollano L, Pepe T and Cortesi ML: Levels of benzo[a]pyrene (BaP) in 'mozzarella di bufala campana' cheese smoked according to different procedures J Agric Food Chem 52: 4452-4455, 2004.

12. Domingo JL: Influence of cooking processes on the concentrations of toxic metals and various organic environmental pollutants in food: A review of the published literature. Crit Rev Food Sci Nutr 51: 29-37, 2011.

13. Guillén MD, Sopelana P and Partearroyo MA: Food as a source of polycyclic aromatic carcinogens. Rev Environ Health 12: 133-146, 1997.

14. Rota M, Bosetti C, Boccia S, Boffetta P and La Vecchia C: Occupational exposures to polycyclic aromatic hydrocarbons and respiratory and urinary tract cancers: An updated systematic review and a meta-analysis to 2014. Arch Toxicol 88: 1479-1490, 2014.

15. Burstyn I, Boffetta P, Heederik D, et al: Mortality from obstructive lung diseases and exposure to polycyclic aromatic hydrocarbons among asphalt workers. Am J Epidemiol 158: 468-478, 2003.

16. Boffetta P, Burstyn I, Partanen T, et al: Cancer mortality among European asphalt workers: An international epidemiological study. I. Results of the analysis based on job titles. Am J Ind Med 43: 18-27, 2003.

17. Iizasa T, Momiki S, Bauer B, Caamano J, Metcalf R, Lechner J, Harris CC and Klein-Szanto AJ: Invasive tumors derived from xenotransplanted, immortalized human cells after in vivo exposure to chemical carcinogens. Carcinogenesis 14: 1789-1794, 1993.

18. Melchiori A, Colacci A, Lollini PL, De Giovanni C, Carlone S, Grilli S, Parodi S and Albini A: Induction of invasive and experimental metastasis potential in BALB/c 3T3 cells by benzo(a)pyrene transformation. Invasion Metastasis 12: 1-11, 1992.

19. Ueng TH, Chang YL, Tsai YY, Su JL, Chan PK, Shih JY, Lee YC, Ma YC and Kuo ML: Potential roles of fibroblast growth factor-9 in the benzo(a)pyrene-induced invasion in vitro and the metastasis of human lung adenocarcinoma. Arch Toxicol 84: 651-660, 2010.

20. Yoshino I, Kometani T, Shoji F, Osoegawa A, Ohba T, Kouso H, Takenaka T, Yohena T and Maehara Y: Induction of epithelial-mesenchymal transition-related genes by benzo[a]pyrene in lung cancer cells. Cancer 110: 369-374, 2007.

21. Wang Y, Zhai W, Wang H, Xia X and Zhang C: Benzo(a)pyrene promotes A549 cell migration and invasion through up-regulating Twist. Arch Toxicol: May 22, 2014 (Epub ahead of print).
22. Martos I, Ferreres F and Tomás-Barberán FA: Identification of flavonoid markers for the botanical origin of Eucalyptus honey. J Agric Food Chem 48: 1498-1502, 2000.

23. Martos I, Ferreres F, Yao L, D'Arcy B, Caffin N and TomásBarberán FA: Flavonoids in monospecific eucalyptus honeys from Australia. J Agric Food Chem 48: 4744-4748, 2000.

24. Yao L, Jiang Y, D'Arcy B, Singanusong R, Datta N, Caffin N and Raymont K: Quantitative high-performance liquid chromatography analyses of flavonoids in Australian Eucalyptus honeys. J Agric Food Chem 52: 210-214, 2004.

25. Geraets L, Moonen HJ, Brauers K, Wouters EF, Bast A and Hageman GJ: Dietary flavones and flavonoles are inhibitors of poly(ADP-ribose)polymerase-1 in pulmonary epithelial cells. J Nutr 137: 2190-2195, 2007.

26. Hsu YL, Uen YH, Chen Y, Liang HL and Kuo PL: Tricetin, a dietary flavonoid, inhibits proliferation of human breast adenocarcinoma mcf-7 cells by blocking cell cycle progression and inducing apoptosis. J Agric Food Chem 57: 8688-8695, 2009.

27. Hsu YL,Hou MF, Tsai EM and Kuo PL: Tricetin, a dietary flavonoid, induces apoptosis through the reactive oxygen species/c-Jun NH2-terminal kinase pathway in human liver cancer cells. J Agric Food Chem 58: 12547-12556, 2010.

28. Casimiro S, Mohammad KS, Pires R, et al: RANKL/RANK/ MMP-1 molecular triad contributes to the metastatic phenotype of breast and prostate cancer cells in vitro. PLoS One 8: e63153, 2013.

29. Krishnan V, Vogler EA, Sosnoski DM and Mastro AM: In vitro mimics of bone remodeling and the vicious cycle of cancer in bone. J Cell Physiol 229: 453-462, 2014.

30. Manenti G, Peissel B, Gariboldi M, et al: A cancer modifier role for parathyroid hormone-related protein. Oncogene 19: 5324-5328, 2000.

31. Wu X, Liu T, Fang O, Leach LJ, Hu X and Luo Z: miR-194 suppresses metastasis of non-small cell lung cancer through regulating expression of BMP1 and p27(kip1). Oncogene 33: 1506-1514, 2014

32. Hernández I, Moreno JL, Zandueta C, Montuenga L and Lecanda F: Novel alternatively spliced ADAM8 isoforms contribute to the aggressive bone metastatic phenotype of lung cancer. Oncogene 29: 3758-3769, 2010.

33. Sone S and Yano S: Molecular pathogenesis and its therapeutic modalities of lung cancer metastasis to bone. Cancer Metastasis Rev 26: 685-689, 2007.

34. Weilbaecher KN, Guise TA and McCauley LK: Cancer to bone: A fatal attraction. Nat Rev Cancer 11: 411-425, 2011.

35. Coleman RE and McCloskey EV: Bisphosphonates in oncology. Bone 49: 71-76, 2011.

36. Hiraki A, Ueoka H, Bessho A, Segawa Y, Takigawa N, Kiura K, Eguchi K, Yoneda T, Tanimoto M and Harada M: Parathyroid hormone-related protein measured at the time of first visit is an indicator of bone metastases and survival in lung carcinoma patients with hypercalcemia. Cancer 95: 1706-1713, 2002.

37. Hsu YL, Tsai EM, Hou MF, Wang TN, Hung JY and Kuo PL: Obtusifolin suppresses phthalate esters-induced breast cancer bone metastasis by targeting parathyroid hormone-related protein. J Agric Food Chem 62: 11933-11940, 2014.

38. Shen $\mathrm{X}$ and Falzon M: PTH-related protein upregulates integrin alpha6beta4 expression and activates Akt in breast cancer cells. Exp Cell Res 312: 3822-3834, 2006.

39. Kitazawa S and Kitazawa R: RANK ligand is a prerequisite for cancer-associated osteolytic lesions. J Pathol 198: 228-236, 2002.

40. Thomas RJ, Guise TA, Yin JJ, Elliott J, Horwood NJ, Martin TJ and Gillespie MT: Breast cancer cells interact with osteoblasts to support osteoclast formation. Endocrinology 140: 4451-4458, 1999.

41. Yasuda H, Shima N, Nakagawa N, et al: A novel molecular mechanism modulating osteoclast differentiation and function. Bone 25: 109-113, 1999 .

42. Jansen ID, Vermeer JA, Bloemen V, Stap J and Everts V: Osteoclast fusion and fission. Calcif Tissue Int 90: 515-522, 2012.

43. Lacey DL, Timms E, Tan HL, et al: Osteoprotegerin ligand is a cytokine that regulates osteoclast differentiation and activation. Cell 93: 165-176, 1998.

44. Jun AY, Kim HJ, Park KK, Son KH, Lee DH, Woo MH and Chung WY: Tetrahydrofurofuran-type lignans inhibit breast cancer-mediated bone destruction by blocking the vicious cycle between cancer cells, osteoblasts and osteoclasts. Invest New Drugs 32: 1-13, 2014. 
45. Bendre MS, Margulies AG, Walser B, et al: Tumor-derived interleukin-8 stimulates osteolysis independent of the receptor activator of nuclear factor-kappaB ligand pathway. Cancer Res 65: 11001-11009, 2005.

46. Hsu YL, Hung JY, Ko YC, Hung CH, Huang MS and Kuo PL: Phospholipase D signaling pathway is involved in lung cancerderived IL-8 increased osteoclastogenesis. Carcinogenesis 31: 587-596, 2010.
47. Lewiecki EM: Safety of long-term bisphosphonate therapy for the management of osteoporosis. Drugs 71: 791-814, 2011.

48. Papapetrou PD: Bisphosphonate-associated adverse events. Hormones (Athens) 8: 96-110, 2009. 